\title{
LARGE DEVIATIONS FOR DENOMINATORS OF CONTINUED FRACTIONS
}

\author{
HIROKI TAKAHASI
}

\begin{abstract}
We give an exponential upper bound on the probability with which the denominator of the $n$th convergent in the regular continued fraction expansion stays away from the mean $\frac{n \pi^{2}}{12 \log 2}$. The exponential rate is best possible, given by an analytic function related to the dimension spectrum of Lyapunov exponents for the Gauss transformation.
\end{abstract}

\section{INTRODUCTION}

Each irrational number $x \in(0,1)$ has the continued fraction expansion

$$
x=\frac{1}{a_{1}+\frac{1}{a_{2}+\cdots}},
$$

where each $a_{i}$ is a positive integer. Let $p_{n}, q_{n}$ be relatively prime positive integers satisfying

$$
\frac{p_{n}}{q_{n}}=\frac{1}{a_{1}+\frac{1}{a_{2}+\cdots+\frac{1}{a_{n}}}} .
$$

Then $p_{n} / q_{n}$ converges to $x$ as $n \rightarrow \infty$, and the rate of this convergence is determined by the growth rate of the denominator $q_{n}$ :

$$
\frac{1}{2 q_{n+1}^{2}} \leq\left|x-\frac{p_{n}}{q_{n}}\right| \leq \frac{1}{q_{n}^{2}}
$$

One important problem in the metric theory of continued fractions is to investigate the limit behavior of $q_{n}$ for typical irrationals. It was Khinchin [9] who proved the existence of an absolute constant $\gamma$ such that $(1 / n) \log q_{n} \rightarrow \gamma$ as $n \rightarrow \infty$ Lebesguea.e. Lévy [10] showed $\gamma=\frac{\pi^{2}}{12 \log 2}$. Hence, for any closed interval $K$ not containing $\gamma$, the Lebesgue measure of the event $\left\{\log q_{n}-\gamma n \in K\right\}$ converges to 0 as $n \rightarrow \infty$. Of interest to know is the rate of this convergence. If it is exponential, namely there is an upper bound of the form $C e^{-\delta n}$ for some $C>0$ and $\delta>0$, then the smallest such $\delta$ would have some intrinsic meaning.

2010 Mathematics Subject Classification. Primary 11A55, 11K50, 37A40, 60F10; Secondary 37A45, 37A50.

Keywords: Diophantine approximation, continued fraction, large deviations. 
Some rates in this convergence are available from central limit theorems. Denote by $\lambda$ the Lebesgue measure restricted to $(0,1)$. Misevičjus [11] showed that

$$
\sup _{\alpha \in \mathbb{R}}\left|\lambda\left\{\frac{\log q_{n}-\gamma n}{\sigma \sqrt{n}} \leq \alpha\right\}-\int_{-\infty}^{\alpha} e^{-\frac{x^{2}}{2}} d x\right|=O\left(\frac{\log n}{\sqrt{n}}\right),
$$

where $\sigma>0$. The results of Morita [12] and Vallée [16] improve the order to $O(1 / \sqrt{n})$. It follows that for every $\alpha<2 \gamma$,

$$
\lambda\left\{\frac{2}{n} \log q_{n} \leq \alpha\right\}=O\left(\frac{1}{\sqrt{n}}\right),
$$

This estimate is far from optimal. From the result of Araújo and Bufetov [1, Theorem B],

$$
\limsup _{n \rightarrow \infty} \frac{1}{n} \log \lambda\left\{\frac{2}{n} \log q_{n} \leq \alpha\right\} \leq I(\alpha),
$$

where the number $I(\alpha)>0$ is defined below. Hence, the convergence takes place at an exponential rate, and the rate can be chosen arbitrarily close to $I(\alpha)$. The aim of this paper is to show that $I(\alpha)$ is the best exponential rate.

We now define $I(\alpha)$ and state our main result. For each $\alpha \in[0, \infty]$ define

$$
L(\alpha)=\left\{x \in(0,1): \liminf _{n \rightarrow \infty} \frac{2}{n} \log q_{n}(x)=\limsup _{n \rightarrow \infty} \frac{2}{n} \log q_{n}(x)=\alpha\right\} .
$$

Put $b(\alpha)=\operatorname{dim}_{H} L(\alpha)$, where $\operatorname{dim}_{H}$ denotes the Hausdorff dimension. Define

$$
I(\alpha)=\alpha(1-b(\alpha)) .
$$

Put $\alpha_{\min }=\log \frac{\sqrt{5}+1}{2}$.

Main Theorem. The following holds:

- for every $n \geq 1$ and every $\alpha \in\left(2 \gamma+\frac{16}{n}, \infty\right)$,

$$
\lambda\left\{\frac{2}{n} \log q_{n} \geq \alpha\right\} \leq C_{\alpha} e^{-I(\alpha) n}
$$

- for every $n \geq 1$ with $\alpha_{\min }<2 \gamma-\frac{16}{n}$ and every $\alpha \in\left(\alpha_{\min }, 2 \gamma-\frac{16}{n}\right)$,

$$
\lambda\left\{\frac{2}{n} \log q_{n} \leq \alpha\right\} \leq C_{\alpha} e^{-I(\alpha) n}
$$

where $C_{\alpha}:=e^{16\left(\left|I^{\prime}(\alpha)\right|+1\right)}$.

The Main Theorem follows from a combination of the multifractal analysis [8, 13] and the thermodynamic formalism [3, 15] associated with the Gauss transformation $T:(0,1] \rightarrow[0,1)$ given by $T x=1 / x-\lfloor 1 / x\rfloor$. It is well-known (see e.g., [7] or Lemmas 2.1 and 2.2) that there exists a constant $C>1$ such that for any irrational $x \in(0,1)$ and $n \geq 1$,

$$
C^{-1} q_{n}^{2}(x) \leq\left|D T^{n}(x)\right| \leq C q_{n}^{2}(x)
$$

These double inequalities permit to translate the analysis of $\log q_{n}$ to that of the Birkhoff sum of the function $\log |D T|$ under the iteration of $T$. The $L(\alpha)$ is the set of irrationals in $(0,1)$ for which the Lyapunov exponent for $T$ is equal to $\alpha$. 


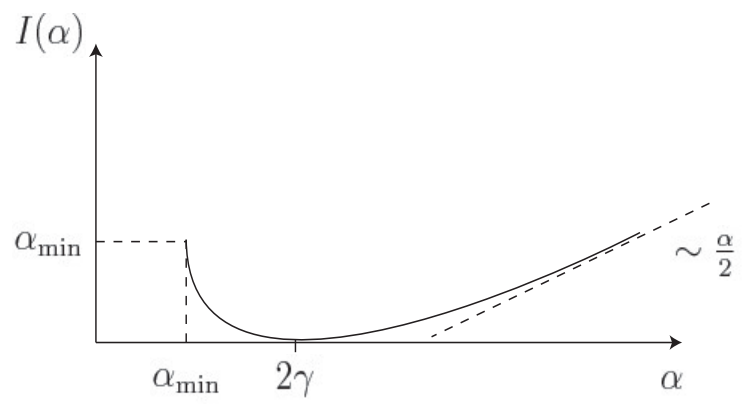

Figure 1. The graph of the function $\alpha \in\left[\alpha_{\min }, \infty\right) \mapsto I(\alpha)$ : $I\left(\alpha_{\min }\right)=\alpha_{\min }, \lim _{\alpha \rightarrow \alpha_{\min }+0} I^{\prime}(\alpha)=-\infty, \lim _{\alpha \rightarrow \infty} I^{\prime}(\alpha)=1 / 2$.

Then $L(\alpha) \neq \emptyset$ holds if and only if $\alpha \in\left[\alpha_{\min }, \infty\right]$, see [8, 13. The function $\alpha \in\left[\alpha_{\min }, \infty\right) \mapsto b(\alpha)$ is known as the dimension spectrum of Lyapunov exponents. It is a non-convex function, analytic on $\left(\alpha_{\min }, \infty\right)$ [8, Theorem 1.3], and $b(\alpha)=1$ holds if and only if $\alpha=2 \gamma$. Hence, $\alpha \in\left(\alpha_{\min }, \infty\right) \mapsto I(\alpha)$ is analytic and $I(\alpha)=0$ holds if and only if $\alpha=2 \gamma$.

The graph of the function $\alpha \in\left[\alpha_{\min }, \infty\right) \mapsto I(\alpha)$ is shown in FIGURE 1. Since $b\left(\alpha_{\min }\right)=0$ by [8, Theorem 1.3], $I\left(\alpha_{\min }\right)=\alpha_{\text {min }}$ holds. Since $I$ is convex by Lemma 2.3. $I^{\prime}(\alpha)$ increases for $\alpha>2 \gamma$. Since $b(\alpha) \rightarrow 1 / 2$ as $\alpha \rightarrow \infty$ by [8, Theorem 1.3], the asymptote exists with slope $1 / 2$.

Since $T$ has infinitely many branches and $\log |D T|$ is unbounded, some finite approximations are necessary for a proof of the Main Theorem. We take finite subsystems, and estimate the exponent of the deviation probabilities in terms of entropy and Lyapunov exponents of invariant probability measures of $T$ supported on the subsystems (Lemma 3.1). Then, using the variational formula for the dimension spectrum [13] we relate the exponent to the function $I$. At the very end we use the convexity and the smoothness of $I$ (in fact, $C^{2}$ is sufficient) to bound error terms arising from the nonlinearity of $T$ and deduce the desired upper bounds.

From [1, Theorem B] the following asymptotic lower bounds hold:

- for every $\alpha \in(2 \gamma, \infty)$,

$$
\liminf _{n \rightarrow \infty} \frac{1}{n} \log \lambda\left\{\frac{2}{n} \log q_{n} \geq \alpha\right\} \geq-I(\alpha) ;
$$

- for every $\alpha \in\left(\alpha_{\min }, 2 \gamma\right)$,

$$
\liminf _{n \rightarrow \infty} \frac{1}{n} \log \lambda\left\{\frac{2}{n} \log q_{n} \leq \alpha\right\} \geq-I(\alpha) .
$$

This means that the exponent $I(\alpha)$ in the Main Theorem is the best possible one. However, the result below on sample means of independent and identically distributed (i.i.d.) random variables leaves the possibility that the upper bounds in the Main Theorem can be improved.

Theorem 1. [2, Theorem 1] Let $\left(X_{n}\right)_{n \geq 1}$ be a sequence of i.i.d. random variables with positive variance with mean 0 . Assume the moment generating function $c(t)=$ 
$\log E\left(e^{t X_{1}}\right)$ is finite on some interval $U$. Let $\alpha>0$ and $t_{\alpha} \in U$ be such that $J(\alpha):=\sup _{t \in U} e^{t \alpha-c(t)}=e^{t_{\alpha} \alpha-c\left(t_{\alpha}\right)}$. Then for every $\alpha>0$,

$$
P\left(S_{n} \geq \alpha n\right)=\frac{b_{n}(1+o(1))}{\sqrt{2 \pi n}} e^{-J(\alpha) n},
$$

where $S_{n}=X_{1}+\cdots+X_{n},\left(b_{n}\right)_{n \geq 1}$ is a sequence of constants and $\inf _{n} b_{n}>0$, $\sup _{n} b_{n}<\infty$.

In [2] it was shown that $\frac{b_{n}(1+o(1))}{\sqrt{2 \pi n}} \leq 1$, and so $P\left(S_{n} \geq \alpha n\right) \leq e^{-J(\alpha) n}$ holds. Such an exponential upper bound was obtained in [5], and follows from Cramér's theorem on the LDP, see [14, pp.26-27]. In the non-i.i.d. case, results for uniformly hyperbolic systems on compact metric spaces with Hölder continuous functions are available [4, Lemma A.1], [17, Theorem 1], which provide upper and lower bounds in agreement with the i.i.d. case in Theorem 1, The bounds in [4, Lemma A.1] are valid only for those $\alpha$ close to the mean.

\section{Preliminary Lemmas}

Before entering the proof of the Main Theorem we need some preliminary lemmas. For each integer $n \geq 1$ denote by $\mathscr{A}^{n}$ the collection of maximal open intervals on which $T^{n}$ is well-defined and continuous. Notice that $q_{n}$ is constant on each element $A \in \mathscr{A}^{n}$. This constant value is denoted by $q_{n}(A)$. For a finite set $\mathscr{B}$ of $\mathscr{A}^{n}$ denote by $[\mathscr{B}]$ the union of all its elements.

Lemma 2.1. For every integer $n \geq 1$ and every $A \in \mathscr{A}^{n}$,

$$
\frac{1}{2} \leq \frac{\lambda(A)}{q_{n}(A)^{-2}}<1
$$

Proof. Assume $n=1$. Each $A \in \mathscr{A}^{1}$ has the form $A=(1 /(k+1), 1 / k)$ for some $k \geq 1$. Then $q_{1}(A)=k$ and so the double inequalities hold. Assume $n \geq 2$ and let $A \in \mathscr{A}^{n}$. For each $i=1, \ldots, n, p_{i}, q_{i}$ are constant on $A$. Denote these constant values by $p_{i}(A)$ and $q_{i}(A)$. By [7, p.18], the endpoints of the interval $A$ are $p_{n}(A) / q_{n}(A)$ and $\left(p_{n}(A)+p_{n-1}(A)\right) /\left(q_{n}(A)+q_{n-1}(A)\right)$. As a consequence,

$$
\lambda(A)=\frac{1}{q_{n}(A)\left(q_{n}(A)+q_{n-1}(A)\right)} .
$$

Since $q_{n}(A)>q_{n-1}(A)$ we obtain the desired double inequalities.

The next lemma used to control the nonlinearity of $T$ can be proved by elementary calculations and hence omitted. See e.g., [6, p.253 Claim] for details.

Lemma 2.2. For every integer $n \geq 1$ and every $A \in \mathscr{A}^{n}$,

$$
\sup _{x, y \in A} \frac{D T^{n}(x)}{D T^{n}(y)} \leq e^{16} .
$$

Write $\phi=-\log |D T|$ and denote by $\mathcal{M}_{\phi}(T)$ the set of $T$-invariant Borel probability measures on $(0,1)$ for which $\phi$ is integrable. For each $\mu \in \mathcal{M}_{\phi}(T)$ denote by $h(\mu)$ the Kolmogorov-Sinal entropy of $\mu$ with respect to $T$, and define $\chi(\mu)=-\int \phi d \mu$. Put $F(\mu)=h(\mu)-\chi(\mu)$. It is known [18] that $\chi(\mu) \geq \alpha_{\min }$ and $F(\mu) \leq 0$. 
Lemma 2.3. For every $\alpha \in\left[\alpha_{\min }, \infty\right)$,

$$
I(\alpha)=\inf \left\{-F(\mu): \mu \in \mathcal{M}_{\phi}(T), \chi(\mu)=\alpha\right\} .
$$

In particular, I is convex.

Proof. Denote the infimum by $\tilde{I}(\alpha)$. Choose a sequence $\left\{\nu_{n}\right\}$ in $\mathcal{M}_{\phi}(T)$ with $\chi\left(\nu_{n}\right)=\alpha$ and $\lim h\left(\nu_{n}\right) / \chi\left(\nu_{n}\right)=b(\alpha)$. Then $\tilde{I}(\alpha) \leq-\lim F\left(\nu_{n}\right)=I(\alpha)$. To show the reverse inequality, choose a sequence $\left\{\mu_{n}\right\}$ in $\mathcal{M}_{\phi}(T)$ with $\chi\left(\mu_{n}\right)=\alpha$ and $-F\left(\mu_{n}\right) \rightarrow \tilde{I}(\alpha)$ as $n \rightarrow \infty$. Fix a measure $\nu \in \mathcal{M}_{\phi}(T)$ with $\chi(\nu)<\alpha$. For each $n$ large enough fix $p_{n} \in(0,1]$ with $\chi\left(p_{n} \mu_{n}+\left(1-p_{n}\right) \nu\right)=\alpha$. Then $\lim p_{n} \rightarrow 1$ and hence $\lim h\left(p_{n} \mu_{n}+\left(1-p_{n}\right) \nu\right)=\alpha-\tilde{I}(\alpha)$. The variational formula in [13] gives

$$
b(\alpha)=\sup \left\{\frac{h(\mu)}{\chi(\mu)}: \mu \in \mathcal{M}_{\phi}(T), \chi(\mu)=\alpha\right\},
$$

and therefore $\alpha^{-1}(\alpha-\tilde{I}(\alpha)) \leq b(\alpha)$, namely $I(\alpha) \leq \tilde{I}(\alpha)$ as required. The convexity of $I$ is a consequence of the affinity of entropy and Lyapunov exponent on measures.

\section{Upper bound With BEST EXPONEnTial RATE}

We are in position to prove the Main Theorem.

Lemma 3.1. Let $n \geq 1$ be an integer and let $\alpha>0$. Let $\mathscr{B}^{n}(\alpha)$ be a nonempty finite subset of $\left\{A \in \mathscr{A}^{n}:(2 / n) \log q_{n}(A) \geq \alpha\right\}$. There exists a measure $\mu \in \mathcal{M}_{\phi}(T)$ such that

$$
\lambda\left[\mathscr{B}^{n}(\alpha)\right] \leq e^{16} e^{F(\mu) n} \quad \text { and } \quad \chi(\mu) \geq \alpha-\frac{16}{n} .
$$

Proof. Put $\widehat{T}=T^{n}$ and $\Lambda=\bigcap_{m=0}^{\infty} \widehat{T}^{-m}\left[\mathscr{B}^{n}(\alpha)\right]$. Then $\Lambda$ is a compact set and $\left.\widehat{T}\right|_{\Lambda}: \Lambda \rightarrow \Lambda$ is continuous. Put $\widehat{\phi}=-\log |D \widehat{T}|$ and fix $y_{0} \in \Lambda$. Lemma 2.2 implies $\sum_{i=0}^{m-1}\left(\widehat{\phi}\left(\widehat{T}^{i}(x)\right)-\widehat{\phi}\left(\widehat{T}^{i}(y)\right)\right) \leq 16$ for every $m \geq 1$, every $x, y \in \Lambda$ such that $\widehat{T}^{i}(x), \widehat{T}^{i}(y)$ belong to the same element of $\mathscr{B}^{n}(\alpha)$ for each $i=0, \ldots, m-1$. The variational principle [3, Lemma 1.20] gives

$$
\sup _{\widehat{\nu} \in \mathcal{M}\left(\left.\widehat{T}\right|_{\Lambda}\right)}\left(h_{\left.\widehat{T}\right|_{\Lambda}}(\widehat{\nu})+\int \widehat{\phi} d \widehat{\nu}\right)=\lim _{m \rightarrow \infty} \frac{1}{m} \log \left(\sum_{x \in\left(\left.\widehat{T}\right|_{\Lambda}\right)^{-m}\left(y_{0}\right)} \exp \sum_{i=0}^{m-1} \widehat{\phi}\left(\widehat{T}^{i}(x)\right)\right),
$$

with $\mathcal{M}\left(\left.\widehat{T}\right|_{\Lambda}\right)$ the space of $\left.\widehat{T}\right|_{\Lambda}$-invariant Borel probability measures endowed with the weak ${ }^{*}$-topology and $h_{\left.\widehat{T}\right|_{\Lambda}}(\widehat{\nu})$ the entropy of $\widehat{\nu} \in \mathcal{M}\left(\left.\widehat{T}\right|_{\Lambda}\right)$ with respect to $\left.\widehat{T}\right|_{\Lambda}$. By Lemma 2.2, $\inf _{[w]} e^{\widehat{\phi}} \geq e^{-16} \lambda[w]$ holds for every $w \in D^{n}$. Hence

$$
\begin{aligned}
\sum_{x \in\left(\left.\widehat{T}\right|_{\Lambda}\right)^{-m}\left(y_{0}\right)} \exp \left(\sum_{i=0}^{m-1} \widehat{\phi}\left(\widehat{T}^{i}(x)\right)\right) & \geq\left(\inf _{y^{\prime} \in \Lambda} \sum_{x \in(\widehat{T} \mid \Lambda)^{-1}\left(y^{\prime}\right)} e^{\widehat{\phi}(x)}\right)^{m} \\
& \geq\left(e^{-16} \lambda\left[\mathscr{B}^{n}(\alpha)\right]\right)^{m} .
\end{aligned}
$$


Taking logs of both sides, dividing by $m$ and plugging the result into the previous inequality gives

$$
\lim _{m \rightarrow \infty} \frac{1}{m} \log \left(\sum_{x \in\left(\left.\widehat{T}\right|_{\Lambda}\right)^{-m}\left(y_{0}\right)} \exp \sum_{i=0}^{m-1} \widehat{\phi}\left(\widehat{T}^{i}(x)\right)\right) \geq \log \lambda\left[\mathscr{B}^{n}(\alpha)\right]-16 .
$$

Plugging this into the previous inequality yields

$$
\sup _{\widehat{\nu} \in \mathcal{M}\left(\left.\widehat{T}\right|_{\Lambda}\right)}\left(h_{\left.\widehat{T}\right|_{\Lambda}}(\widehat{\nu})+\int \widehat{\phi} d \widehat{\nu}\right) \geq \log \lambda\left[\mathscr{B}^{n}(\alpha)\right]-16 .
$$

Since $\mathcal{M}\left(\left.\widehat{T}\right|_{\Lambda}\right)$ is compact and $\mathcal{M}\left(\left.\widehat{T}\right|_{\Lambda}\right) \ni \widehat{\nu} \mapsto h_{\left.\widehat{T}\right|_{\Lambda}}(\widehat{\nu})+\int \widehat{\phi} d \widehat{\nu}$ is upper semicontinuous, there exists a measure $\widehat{\mu} \in \mathcal{M}\left(\left.\widehat{T}\right|_{\Lambda}\right)$ which attains this supremum. The measure $\mu=(1 / n) \sum_{i=0}^{n-1} \widehat{\mu} \circ T^{-i}$ is in $\mathcal{M}_{\phi}(T)$. From the second inequality in Lemma 2.1 and Lemma 2.2 , $\inf _{\left[\mathscr{B}^{n}(\alpha, c)\right]} \log |D \widehat{T}| \geq \alpha n-16$ holds. Hence $\chi(\mu)=$ $(1 / n) \int \log |D \widehat{T}| d \widehat{\mu} \geq \alpha-16 / n$ as required.

Proof of the Main Theorem. Let $n \geq 1$ be an integer. We concentrate on the case $\alpha \in\left(2 \gamma+\frac{16}{n}, \infty\right)$ since the case $\alpha \in\left(\alpha_{\min }, 2 \gamma-\frac{16}{n}\right)$ is identical with the obvious modifications of statements. Denote by $\lambda_{n}$ the distribution of $(2 / n) \log q_{n}$. For each $c>1$ choose a finite subset $\mathscr{B}^{n}(\alpha, c)$ of $\mathscr{A}^{n}$ such that $\lambda_{n}([\alpha, \infty)) \leq$ $c \lambda\left[\mathscr{B}^{n}(\alpha, c)\right]$. By Lemma 3.1 there exists $\mu \in \mathcal{M}_{\phi}(T)$ which satisfies $\lambda\left[\mathscr{B}^{n}(\alpha, c)\right] \leq$ $e^{16} \exp (F(\mu) n)$ and $\chi(\mu) \geq \alpha-16 / n$. Therefore

$$
\begin{aligned}
\lambda_{n}([\alpha, \infty)) & \leq c e^{16} \exp (F(\mu) n) \\
& \leq c e^{16} \exp \left(\sup \left\{F(\mu): \mu \in \mathcal{M}_{\phi}(T), \chi(\mu) \geq \alpha-\frac{16}{n}\right\}\right) \\
& \leq c e^{16} e^{-\inf _{\beta \in[\alpha-16 / n, \infty)} I(\beta)} \\
& =c e^{16} e^{-I(\alpha-16 / n) n} \\
& \leq c e^{16\left(I^{\prime}(\alpha)+1\right)} e^{-I(\alpha) n} .
\end{aligned}
$$

For the last inequality we have used the convexity and the smoothness of $I$. Since $c>1$ is arbitrary, we obtain $\lambda_{n}([\alpha, \infty)) \leq C_{\alpha} e^{-I(\alpha) n}$ as required.

Acknowledgments. This research was partially supported by the Grant-in-Aid for Young Scientists (A) of the JSPS $15 \mathrm{H} 05435$ and the Grant-in-Aid for Scientific Research (B) of the JSPS 16KT0021.

\section{REFERENCES}

[1] V. Araújo, A. I. Bufetov, A large deviations bound for the Teichmüller flow on the moduli space of abelian differentials, Ergodic Theory and Dynamical Systems 31 (2011) 1043-1071.

[2] R. R. Bahadur, R. Ranga Rao, On deviations of the sample mean, Ann. Math. Statist. 31 (1960) 1015-1027.

[3] R. Bowen, Equilibrium states and the ergodic theory of Anosov diffeomorphisms, Second revised edition. Lecture Notes in Mathematics, 470 (2008) Springer-Verlag, Berlin

[4] J.-R. Chazottes, P. Collet, Almost-sure central limit theorems and the Erdös-Rényi law for expanding maps of the interval. Ergodic Theory and Dynamical Systems 25 (2005) 419-441. 
[5] H. Chernoff, A measure of asymptotic efficiency for tests of hypothesis based on the sum of observations. Ann. Math. Statist. 23 (1952) 493-507.

[6] D. Fiebig, U.-R. Fiebig, M. Yuri, Pressure and equilibrium states for countable state Markov shifts. Israel J. Math. 131 (2002) 221-257.

[7] M. Iosifescu, M, C. Kraaikamp, Metric theory of continued fractions. Kluwer Academic, Dordrecht, 2002

[8] M. Kesseböhmer, B. O. Stratmann, A multifractal analysis for Stern-Brocot intervals, continued fractions and Diophantine growth rates. J. reine angel. Math. 605 (2007) 133-163.

[9] A. Y. Khinchin, Metrische Kettenbruchprobleme. Composito Math. 1 (1935) 361-382.

[10] P. Lévy, Théorie de l'addition des variables aléatoires. Paris, Gauthier-Villars (1937)

[11] G. Misevičjus, Estimate of the reminder term in the limit theorem for the denominators of continued fractions. Lithuanian Math. J. 21 (1981) 245-253.

[12] T. Morita, Local limit theorem and distribution of periodic orbits of Lasota-Yorke transformations with infinite Markov partition. J. Math. Soc. Japan 46 (1994) 309-343.

[13] M. Pollicott, B. Weiss, Multifractal analysis of Lyapunov exponent for continued fraction and Manneville-Pomeau transformations and applications to Diophantine Approximation. Commun. Math. Phys. 207 (1999) 145-171.

[14] F. Rassoul-Agha and T. Seppäläinen, A course on large deviations with an introduction to Gibbs measures, Graduate Studies in Mathematics, 162. American Mathematical Society, Providence, RI, 2015.

[15] D. Ruelle, Thermodynamic formalism. The mathematical structures of classical equilibrium statistical mechanics. Second edition. Cambridge University Press (2004)

[16] B. Vallée, Opérateurs de Ruelle-Mayer généralisés et analyse des algorithmes d'Euclide et de Gauss. Acta Arith. 81 (1997) 101-144.

[17] S. Waddington, Large deviation asymptotics for Anosov flows. Ann. Inst. Henri Poincaré 13 (1996) 445-484.

[18] P. Walters, Invariant measures and equilibrium states for some mappings which expand distances. Trans. Amer. Math. Soc. 236 (1978) 121-153.

Keio Institute of Pure and Applied Sciences (KiPAS), Department of Mathematics, Keio University, Yokohama, 223-8522, JAPAN

E-mail address: hiroki@math.keio.ac.jp

URL: http://www.math.keio.ac.jp/ ^hiroki/ 